\title{
Bacteriophages: A Revisited Strategy for the Treatment of Severe Bacterial Infections
}

\author{
Roberto Badaro \\ Deputy Editor; Director Health Institute of Technologies SENAI CIMATEC; Salvador, Bahia, Brazil
}

Bacteriophages are viruses that infect and parasitize bacteria [1]. Phages were discovered by Frederick Willian Twort in 1915, an England microbiologist, based at the St. Thomas Hospital in London, during his tentative to grow a virus in culture media. He found water looking areas in a bacteria culture tubes that later he correlated with some virus that made impossible colonies of bacteria grow on any medium. He had inoculated an agar medium with some fluid (unfiltered), commonly used for smallpox vaccinations [2]. He noticed that although the vaccinia virus did not grow, a bacteria (micrococcus) did grow. The bacteria, however, appeared to be affected with some disease - inoculated agar tubes often showed watery-looking areas, and in cultures that grew micrococci, it was found that some of these colonies could not be subcultured, but if kept, they became glassy and transparent.

In retrospect, this phenomenon, which came to be known as the glassy transformation of Twort, seems exceedingly strange, but indeed was the bacteriophage. An observed agent with Brownian motion present in unfilterable, heatlabile culture media activity against Vibrio cholerae, was independently investigated by a French microbiologist, Felix d'Herelle, in 1917. D'Herelle noticed an anomaly shown by some cultures of the coccobacillus that consisted of clear spots, quite circular, two or three millimeters in diameter, speckling the cultures grown on agar of patients with isolates from the bloody stools dysentery bacillus. Still, the spreading on agar of

Received on 26 August 2019; revised 27 September 2019. Address for correspondence: Dr. Roberto Badaró. ITSSENAI-CIMATEC. Avenida Orlando Gomes, 1845, Piatã. CEP: 41.650-010. Salvador, Bahia, Brazil. E-mail: badaro@ fieb.org.br.

J Bioeng. Biotech. Appl. Health 2019;2(3):78-79.

(C) 2019 by SENAI CIMATEC. All rights reserved. a broth culture, to which had been added a filtrate from the feces spread, was devoid of all growth and lead him to conclude that what caused that clear spot was, in fact, an invisible microbe, a filterable virus, but a virus parasitic on bacteria. Later, called bacteria eaters, the bacteriophages [1].

From 1920 to 1940, prior penicillin discovery, Phage therapy was the only one antimicrobial available treatment in western Europe [3].

Bacteriophage multiplies using two different mechanisms: a lytic cycle that determines the lysis of the infected bacteria and the lysogenic cycle that depending on whether they contain integrase and repressor genes. Those that contain these genes can become integrated into the bacterial chromosome and replicate with the bacterium without killing it. They are termed "temperate" or "lysogenic" phages (as opposed to "lytic" phages that lack this characteristic) [4]. Phages replication have and important implications for their therapeutic application. Virulent or obligate lytic phages infect and quickly kill their bacterial host cell, whereas temperate or lysogenic phages may either stably integrate into their host's genome or enter into the lytic life cycle [3]. Each phage recognizes a specific molecular motif on the surface of its bacterial target. Once bound to their receptors, they pin themselves to the membrane and inject phage DNA into the interior of the bacterium. Bacterial metabolism is taken over by the phage, and the bacteria make 100-1,000 phage copies as well as phage-specified lysin proteins that blow up the bacterial target. Progeny phage then attacks additional susceptible bacteria in the population [5]. These types of phages can pick up bacterial genes when they are reactivated and move bacterial resistance genes, pathogenicity genes to other bacteria. Besides, they create defensive "repressor" molecules that inhibit the ability of new phages to attack the bacterium into which they 
are integrated. Natural phages for certain types of bacteria are almost exclusively lysogenic (e.g., mycobacterial phages). Each phage is specific to a particular bacterial genus or species [6].

The current increase in the incidence of antibiotic resistance in human bacteria has favored the study of phages as a therapeutic alternative (phage therapy) [4]. Phage therapy is defined as the administration of virulent phages directly to a patient to lyse the bacterial pathogen that is causing a clinically severe infection [7]. Previous studies have shown the efficacy that revisiting phages therapy patients with severe antibiotic resistance infections were successfully treated [8]. Today it is definitively an alternative treatment for multidrug resistance microorganisms (MDR) that antibiotic failure [9]. Bacteriophage therapies reenter clinical trials [10]. Different clinical trials are underway to establish the safety, reactogenicity, and therapeutic efficacy of multiple phages. As active elements, phages must undergo rigorous quality controls to ensure the absence of undesirable effects. The bacterial lysis that they cause is of magnitude inferior to what antibiotics do. The future problem must be solved as the possibility of using mixtures of several phages, establish the ideal route of administration and modify them genetically to deactivate bacterial resistance genes, thus antibiotic recovery sensitivity of MDR organisms.

\section{References}

1. Watts, G. Phage therapy: revival of the bygone antimicrobial. Lancet. 2017;390(10112):2539-2540.

2. Duckworth, D. Who Discovered Bacteriophage? Bacteriological Reviews. 1976;40(4):793-802.

3. Gordillo Altamirano, F.L., Barr, J.J. Phage therapy in the tostantibiotic era. Clin Microbiol Rev. 2019;32(2).

4. Reina, J., Reina, N. Phage therapy, an alternative to antibiotic therapy? Rev Esp Quim. 2018;31(2):101-104.

5. Labrie, S.J., Samson, J.E., Moineau, S. Bacteriophage resistance mechanisms. Nat Rev Microbiol. 2010;8(5):317-327.

6. Sharma, S., Chatterjee, S., Datta, S., Prasad, R., Dubey, D., Prasad, R.K., Vairale, M.G. Bacteriophages and its applications: an overview. Folia Microbiol (Praha) 2017;62(1):17-55.

7. Viertel, T.M., Ritter, K., Horz, H.P. Viruses versus bacteria-novel approaches to phage therapy as a tool against multidrug-resistant pathogens. J Antimicrob Chemother. 2014;69(9):2326-2336.

8. Schooley, R.T., Biswas, B., Gill, J.J., HernandezMorales, A., Lancaster, J., Lessor, L., Barr. J.J., Reed, S.L., Rohwer, F., Benler, S., et al. Development and use of personalized bacteriophage-based therapeutic cocktails to treat a patient with a disseminated resistant Acinetobacter baumannii infection. Antimicrob Agents Chemother. 2017;61(10).

9. Kakasis, A., Panitsa, G. Bacteriophage therapy as an alternative treatment for human infections. A comprehensive review. Int $\mathrm{J}$ Antimicrob Agents. 2019;503(1):16-21.

10 Kingwell K. Bacteriophage therapies re-enter clinical trials. Nat Rev Drug Discov. 2015;14(8):515-516. 